\title{
Implementation of Novel Method in Curve Interpolation
}

\author{
[Dariusz Jacek Jakóbczak]
}

\begin{abstract}
Applied science and mechanics need mathematical methods for $2 \mathrm{D}$ processes modeling using the set of data points. A novel method of Hurwitz-Radon Matrices (MHR) is used in 2D curve modeling. Proposed method is based on the family of Hurwitz-Radon matrices which possess columns composed of orthogonal vectors. Two-dimensional process is modeled via different functions: sine, cosine, tangent, logarithm, exponent, arc sin, arc cos, arc tan and power function.
\end{abstract}

Keywords - process modeling, Hurwitz-Radon matrices, coefficient of MHR method, mathematical modeling

\section{Introduction}

2D process modeling is a critical part in many applications of applied science. Thus mechanics and numerical methods have a problem: how to modeled the curve of data [1,2] via discrete set of two-dimensional points? Also subject of 2D curve interpolation and mathematical shape description is still opened $[3,4]$. The author wants to approach a problem of process modeling by characteristic points and not limited to closed curves, but also working on open curves. Proposed method relies on functional modeling of curve points situated between the basic set of the nodes. The functions that are used in calculations represent whole family of elementary functions: trigonometric, cyclometric, logarithmic, exponential and power function. Nowadays methods apply mainly polynomial functions, for example Bernstein polynomials in Bezier curves, splines and NURBS [5]. Numerical methods for data interpolation are based on polynomial or trigonometric functions, for example Lagrange, Newton, Aitken and Hermite methods. These methods have some weak sides [6] and are not sufficient for object modeling in the situations when the shape cannot be build by polynomials or trigonometric functions. Also trigonometric basis functions in Fourier Series Shape Models are not appropriate for describing all curves. In this paper discussed approach is not limited to closed curves. Proposed mathematical 2D processes modeling is the modeling via any elementary functions. The author presents novel method of flexible modeling of 2D data.

This paper takes up new method of two-dimensional process modeling by using a family of Hurwitz-Radon matrices. The method of Hurwitz-Radon Matrices (MHR) requires minimal assumptions about process. The only information is the set of at least two nodes. Proposed method of Hurwitz-Radon Matrices (MHR) is applied in curve modeling via different coefficients: sinusoidal, cosinusoidal,

Dariusz Jacek Jakóbczak

Technical University of Koszalin

Poland tangent, logarithmic, exponential, arc sin, arc cos, arc tan or power. Function for coefficient calculations is chosen individually at each modeling and it depends on initial requirements and process specifications. Data of the process are represented by succeeding points $\left(x_{i}, y_{i}\right) \in \boldsymbol{R}^{\mathbf{2}}$ as follows in MHR method:

1. MHR version with no matrices $(N=1)$ needs 2 nodes or more;

2. At least five nodes $\left(x_{1}, y_{1}\right),\left(x_{2}, y_{2}\right),\left(x_{3}, y_{3}\right),\left(x_{4}, y_{4}\right)$ and $\left(x_{5}, y_{5}\right)$ if MHR method is implemented with matrices of dimension $N=2$;

3. For better modeling nodes ought to be settled at key points of the curve, for example local minimum or maximum and at least one point between two successive local extrema.

Condition 2 is connected with important features of MHR method: MHR version with matrices of dimension $N=2$ needs at least five nodes, MHR version with matrices of dimension $N=4$ needs at least nine nodes and MHR version with matrices of dimension $N=8$ needs at least 17 nodes. Condition 3 means for example the highest point of the object in a particular orientation, convexity changing or curvature extrema. So this paper wants to answer the question: how to model the $2 \mathrm{D}$ process for the set of data points?

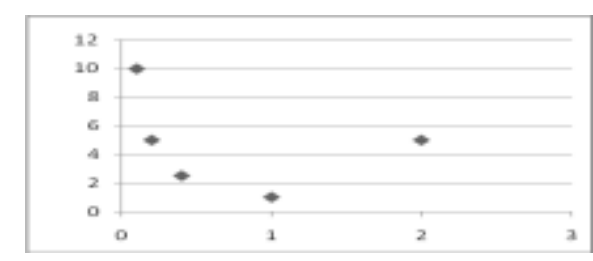

Figure 1. Nodes of the process before 2D modeling.

Coefficients for mathematical 2D process modeling are computed via individual features of data using power function, sine, cosine, tangent, logarithm, exponent or arc sin, arc cos, arc tan or others.

\section{Application of MHR}

The method of Hurwitz - Radon Matrices (MHR), described in this paper, is computing points between two successive nodes of the curve. Points are interpolated and parameterized for real number $\alpha \in[0 ; 1]$ in the range of two successive nodes. MHR calculations are introduced with square matrices of dimension $N=1,2,4$ or 8 . Matrices $A_{i}, i=$ $1,2 \ldots m$ satisfying

$$
A_{j} A_{k}+A_{k} A_{j}=0, A_{j}^{2}=-I \text { for } j \neq k ; j, k=1,2 \ldots m
$$


are called a family of Hurwitz - Radon matrices, discussed by Adolf Hurwitz and Johann Radon separately in 1923. A family of Hurwitz - Radon (HR) matrices [7] are skew-symmetric $\left(A_{i}{ }^{\mathrm{T}}=-A_{i}, A_{i}^{-1}=-A_{i}\right)$ and only for dimension $N=1,2,4$ or 8 the family of HR matrices consists of $N-1$ matrices. So far HR matrices have found applications in Space-Time Block Coding (STBC) [8] and orthogonal design [9], in signal processing [10] and Hamiltonian Neural Nets [11].

How coordinates of data points are applied in 2D process modeling? If data points have the set of following nodes $\left\{\left(x_{i}, y_{i}\right), i=1,2, \ldots, n\right\}$ then HR matrices combined with the identity matrix $I_{N}$ are used to build the orthogonal Hurwitz Radon Operator (OHR). For point $p_{1}=\left(x_{1}, y_{1}\right)$ OHR of dimension $N=1$ is represented by matrix (real number) $M_{1}$ :

$$
M_{1}\left(p_{1}\right)=\frac{1}{x_{1}^{2}}\left[x_{1} \cdot y_{1}\right]=\frac{y_{1}}{x_{1}}
$$

For points $p_{1}=\left(x_{1}, y_{1}\right)$ and $p_{2}=\left(x_{2}, y_{2}\right)$ OHR of dimension $N=2$ is build via matrix $M_{2}$ :

$$
M_{2}\left(p_{1}, p_{2}\right)=\frac{1}{x_{1}^{2}+x_{2}^{2}}\left[\begin{array}{ll}
x_{1} y_{1}+x_{2} y_{2} & x_{2} y_{1}-x_{1} y_{2} \\
x_{1} y_{2}-x_{2} y_{1} & x_{1} y_{1}+x_{2} y_{2}
\end{array}\right] \text {. }
$$

For points $p_{1}=\left(x_{1}, y_{1}\right), p_{2}=\left(x_{2}, y_{2}\right), p_{3}=\left(x_{3}, y_{3}\right)$ and $p_{4}=\left(x_{4}, y_{4}\right) \mathrm{OHR}$ $M_{4}$ of dimension $N=4$ is introduced:

$M_{4}\left(p_{1}, p_{2}, p_{3}, p_{4}\right)=\frac{1}{x_{1}^{2}+x_{2}^{2}+x_{3}^{2}+x_{4}^{2}}\left[\begin{array}{cccc}u_{0} & u_{1} & u_{2} & u_{3} \\ -u_{1} & u_{0} & -u_{3} & u_{2} \\ -u_{2} & u_{3} & u_{0} & -u_{1} \\ -u_{3} & -u_{2} & u_{1} & u_{0}\end{array}\right]$ (3)

where

$u_{0}=x_{1} y_{1}+x_{2} y_{2}+x_{3} y_{3}+x_{4} y_{4}, \quad u_{1}=-x_{1} y_{2}+x_{2} y_{1}+x_{3} y_{4}-x_{4} y_{3}$, $u_{2}=-x_{1} y_{3}-x_{2} y_{4}+x_{3} y_{1}+x_{4} y_{2}, \quad u_{3}=-x_{1} y_{4}+x_{2} y_{3}-x_{3} y_{2}+x_{4} y_{1}$.

For nodes $p_{1}=\left(x_{1}, y_{1}\right), p_{2}=\left(x_{2}, y_{2}\right), \ldots$ and $p_{8}=\left(x_{8}, y_{8}\right)$ OHR $M_{8}$ of dimension $N=8$ is constructed [12] similarly as (1)-(3):

$M_{8}\left(p_{1}, p_{2} \ldots p_{8}\right)=\frac{1}{\sum_{i=1}^{8} x_{i}^{2}}\left[\begin{array}{cccccccc}u_{0} & u_{1} & u_{2} & u_{3} & u_{4} & u_{5} & u_{6} & u_{7} \\ -u_{1} & u_{0} & u_{3} & -u_{2} & u_{5} & -u_{4} & -u_{7} & u_{6} \\ -u_{2} & -u_{3} & u_{0} & u_{1} & u_{6} & u_{7} & -u_{4} & -u_{5} \\ -u_{3} & u_{2} & -u_{1} & u_{0} & u_{7} & -u_{6} & u_{5} & -u_{4} \\ -u_{4} & -u_{5} & -u_{6} & -u_{7} & u_{0} & u_{1} & u_{2} & u_{3} \\ -u_{5} & u_{4} & -u_{7} & u_{6} & -u_{1} & u_{0} & -u_{3} & u_{2} \\ -u_{6} & u_{7} & u_{4} & -u_{5} & -u_{2} & u_{3} & u_{0} & -u_{1} \\ -u_{7} & -u_{6} & u_{5} & u_{4} & -u_{3} & -u_{2} & u_{1} & u_{0}\end{array}\right]$

where

$$
\underline{u}=\left[\begin{array}{cccccccc}
y_{1} & y_{2} & y_{3} & y_{4} & y_{5} & y_{6} & y_{7} & y_{8} \\
-y_{2} & y_{1} & -y_{4} & y_{3} & -y_{6} & y_{5} & y_{8} & -y_{7} \\
-y_{3} & y_{4} & y_{1} & -y_{2} & -y_{7} & -y_{8} & y_{5} & y_{6} \\
-y_{4} & -y_{3} & y_{2} & y_{1} & -y_{8} & y_{7} & -y_{6} & y_{5} \\
-y_{5} & y_{6} & y_{7} & y_{8} & y_{1} & -y_{2} & -y_{3} & -y_{4} \\
-y_{6} & -y_{5} & y_{8} & -y_{7} & y_{2} & y_{1} & y_{4} & -y_{3} \\
-y_{7} & -y_{8} & -y_{5} & y_{6} & y_{3} & -y_{4} & y_{1} & y_{2} \\
-y_{8} & y_{7} & -y_{6} & -y_{5} & y_{4} & y_{3} & -y_{2} & y_{1}
\end{array}\right] \cdot\left[\begin{array}{c}
x_{1} \\
x_{2} \\
x_{3} \\
x_{4} \\
x_{5} \\
x_{6} \\
x_{7} \\
x_{8}
\end{array}\right]
$$

and $\underline{u}=\left(u_{0}, u_{1}, \ldots, u_{7}\right)^{\mathrm{T}}$. OHR operators $M_{N}$ (1)-(4) satisfy the condition of interpolation

$$
M_{N} \cdot \mathbf{x}=\mathbf{y}
$$

for $\mathbf{x}=\left(x_{1}, x_{2} \ldots, x_{N}\right)^{\mathrm{T}} \in \boldsymbol{R}^{N}, \mathbf{x} \neq \mathbf{0}, \mathbf{y}=\left(y_{1}, y_{2} \ldots, y_{N}\right)^{\mathrm{T}} \in \boldsymbol{R}^{N}$ and $N=1,2,4$ or 8 .

\section{A. Modeling Functions}

Coordinates of points settled between the nodes are computed [13] using presented MHR method [14]. Each real number $c \in[a ; b]$ is calculated by a convex combination $c=\alpha \cdot a+(1-\alpha) \cdot b$ for

$$
\alpha=\frac{b-c}{b-a} \in[0 ; 1]
$$

The weighted OHR matrix operator $M$ of dimension $N=1,2$, 4 or 8 is build:

$$
M=\gamma \cdot A+(1-\gamma) \cdot B
$$

The OHR matrix $A$ is constructed (1)-(4) by every second point $p_{1}=\left(x_{1}=a, y_{1}\right), p_{3}=\left(x_{3}, y_{3}\right), \ldots$ and $p_{2 N-1}=\left(x_{2 N-1}, y_{2 N-1}\right)$ :

$$
A=M_{N}\left(p_{1}, p_{3, \ldots,} p_{2 N-1}\right) \text {. }
$$

The OHR matrix $B$ is computed (1)-(4) by data $p_{2}=\left(x_{2}=b, y_{2}\right)$, $p_{4}=\left(x_{4}, y_{4}\right), \ldots$ and $p_{2 N}=\left(x_{2 N}, y_{2 N}\right)$ :

$$
B=M_{N}\left(p_{2}, p_{4, \ldots,} p_{2 N}\right) .
$$

Vector of first coordinates $C$ is defined for

$$
c_{i}=\alpha \cdot x_{2 i-1}+(1-\alpha) \cdot x_{2 i}, \quad i=1,2, \ldots, N
$$

and $C=\left[c_{1}, c_{2}, \ldots, c_{N}\right]^{\mathrm{T}}$. The formula to calculate second coordinates $y\left(c_{i}\right)$ is similar to the interpolation formula (5):

$$
Y(C)=M \cdot C
$$

where $Y(C)=\left[y\left(c_{1}\right), y\left(c_{2}\right), \ldots, y\left(c_{N}\right)\right]^{\mathrm{T}}$. So modeled value of $y\left(c_{i}\right)$ depends on two, four, eight or sixteen $(2 N)$ successive nodes. For example $N=1$ results in computations without matrices:

$$
A=M_{1}\left(p_{1}\right)=\frac{y_{1}}{x_{1}}, B=M_{1}\left(p_{2}\right)=\frac{y_{2}}{x_{2}}, C=c_{1}=\alpha \cdot x_{1}+(1-\alpha) \cdot x_{2},
$$


Proc. of the Intl. Conf. on Advances in Computing, Control and Networking - ACCN 2015.

Copyright $($ Institute of Research Engineers and Doctors, USA .All rights reserved.

ISBN: 978-1-63248-038-5 doi: 10.15224/ 978-1-63248-038-5-02

$$
\begin{gathered}
Y(C)=y\left(c_{1}\right)=\left(\gamma \frac{y_{1}}{x_{1}}+(1-\gamma) \frac{y_{2}}{x_{2}}\right) \cdot c_{1}, \\
y\left(c_{1}\right)=\alpha \cdot \gamma \cdot y_{1}+(1-\alpha)(1-\gamma) y_{2}+\gamma(1-\alpha) \frac{y_{1}}{x_{1}} x_{2}+\alpha(1-\gamma) \frac{y_{2}}{x_{2}} x_{1}
\end{gathered}
$$

Key question is dealing with coefficient $\gamma$ in (7). Basic MHR version means $\gamma=\alpha$ and then (10):

$$
y\left(c_{1}\right)=\alpha^{2} \cdot y_{1}+(1-\alpha)^{2} y_{2}+\alpha(1-\alpha)\left(\frac{y_{1}}{x_{1}} x_{2}+\frac{y_{2}}{x_{2}} x_{1}\right) .
$$

Formula (11) differs from linear interpolation: $y(c)=\alpha \cdot y_{1}+(1-\alpha) y_{2}$. MHR is not a linear interpolation. For $N=2$ equation (10) turns to parameterization:

$$
y\left(c_{1}\right)=\alpha \cdot \gamma \cdot y_{1}+(1-\alpha)(1-\gamma) y_{2}+\gamma(1-\alpha) r_{1}+\alpha(1-\gamma) r_{2}
$$

where

$$
r_{1}=\frac{1}{x_{1}^{2}+x_{3}^{2}}\left(x_{1} x_{2} y_{1}+x_{2} x_{3} y_{3}+x_{3} x_{4} y_{1}-x_{1} x_{4} y_{3}\right)
$$

and

$$
r_{2}=\frac{1}{x_{2}{ }^{2}+x_{4}{ }^{2}}\left(x_{1} x_{2} y_{2}+x_{1} x_{4} y_{4}+x_{3} x_{4} y_{2}-x_{2} x_{3} y_{4}\right) \text {. }
$$

Each process requires specific parameter $\gamma(7)$ and $\gamma$ depends on parameter $\alpha \in[0 ; 1]: \gamma=\mathrm{F}(\alpha), \mathrm{F}:[0 ; 1] \rightarrow[0 ; 1], \quad \mathrm{F}(0)=0$, $\mathrm{F}(1)=1$ and $\mathrm{F}$ is strictly monotonic. Coefficient $\gamma$ is calculated using different functions (power, sinus, cosine, tangent, logarithm, exponent, arc sin, arc cos, arc tan or others) and choice of function is connected with process specifications. Different values of coefficient $\gamma$ are connected with implemented functions for real parameter $k>0$ :

1. power function $\gamma=\alpha^{k}$

2. sine $\gamma=\sin \left(\alpha^{k} \cdot \pi / 2\right)$ or $\gamma=\sin ^{k}(\alpha \cdot \pi / 2)$

3. cosine $\gamma=1-\cos \left(\alpha^{k} \cdot \pi / 2\right)$ or $\gamma=1-\cos ^{k}(\alpha \cdot \pi / 2)(15)$

4. tangent $\gamma=\tan \left(\alpha^{k} \cdot \pi / 4\right)$ or $\gamma=\tan ^{k}(\alpha \cdot \pi / 4)$

5. logarithm $\gamma=\log _{2}\left(\alpha^{k}+1\right)$ or $\gamma=\log _{2}{ }^{k}(\alpha+1)$

6. exponent

$$
\gamma=\left(\frac{a^{\alpha}-1}{a-1}\right)^{k}, a>0 \text { and } a \neq 1
$$

For $k=1$ and $a=2: \quad \gamma=2^{\alpha}-1$.

7. $\operatorname{arcsine} \gamma=2 / \pi \cdot \arcsin \left(\alpha^{k}\right)$ or $\gamma=(2 / \pi \cdot \arcsin \alpha)^{k}$

8. arccosine $\gamma=1-2 / \pi \cdot \arccos \left(\alpha^{k}\right)$ or $\gamma=1-(2 / \pi \cdot \arccos \alpha)^{k}$

9. $\operatorname{arctangent} \gamma=4 / \pi \cdot \arctan \left(\alpha^{k}\right)$ or $\gamma=(4 / \pi \cdot \arctan \alpha)^{k}$
What is very important, functions used in $\gamma$ calculations (13)-(21) are strictly monotonic for $\alpha \in[0 ; 1]$, because $\gamma \in[0 ; 1]$ too. Choice of modeling function depends on data specifications and individual requirements. Fixing of unknown coordinates for curve points using (6)-(9) is called by author the method of Hurwitz - Radon Matrices (MHR) [15]. Here are five steps of MHR mathematical 2D process modeling:

Step 1: Choice of nodes at key points.

Step 2: Fixing the dimension of matrices $N=1,2,4$ or $8: N=$ 1 is the most universal for calculations (it needs only two successive nodes to compute unknown point between them) and it has the lowest computational costs (10); MHR with $N=$ 2 uses four successive nodes to compute unknown coordinate; MHR version for $N=4$ applies eight successive nodes to get unknown point and MHR with $N=8$ needs sixteen successive nodes to calculate unknown coordinate (it has the biggest computational costs).

Step 3: Choice of function $\gamma=\mathrm{F}(\alpha)$ : basic MHR for $\gamma=\alpha$.

Step 4: Determining values of $\alpha: \alpha=0.1,0.2 \ldots 0.9$ or 0.01 , $0.02 \ldots 0.99$ or others.

Step 5: The computations (9).

These five steps can be treated as the algorithm of MHR method of 2D curve modeling and interpolation (6)-(9).

\section{Application of MHR Data Reconstruction}

Data nodes: $(0.1 ; 10),(0.2 ; 5),(0.4 ; 2.5),(1 ; 1)$ and $(2 ; 5)$ from Fig.1 are used in some examples of MHR 2D process modeling with different $\gamma$. Points of the object are calculated with matrices of dimension $N=2$ and $\alpha=0.1,0.2, \ldots, 0.9$.

Example 1

Sinusoidal modeling with $\gamma=\sin (\alpha \cdot \pi / 2)$.

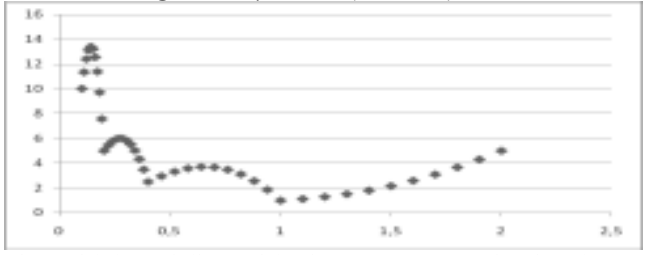

Figure 2. Sinusoidal modeling with nine reconstructed points between nodes

Example 2

Tangent modeling for $\gamma=\tan (\alpha \cdot \pi / 4)$.

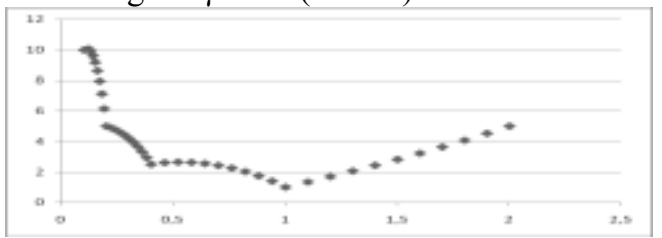

Figure 3. Tangent modeling with nine interpolated points between nodes.

Example 3

Power function interpolation for $\gamma=\alpha^{k}$ and $k=2.1205$. 
Proc. of the Intl. Conf. on Advances in Computing, Control and Networking - ACCN 2015.

Copyright $($ Institute of Research Engineers and Doctors, USA .All rights reserved.

ISBN: 978-1-63248-038-5 doi: 10.15224/ 978-1-63248-038-5-02

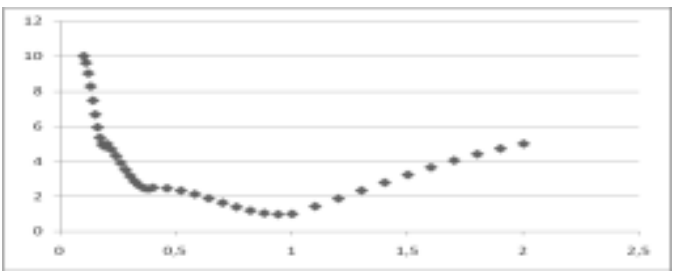

Figure 4. Power function modeling with nine recovered object points between nodes.

Example 4

Logarithmic modeling with $\gamma=\log _{2}\left(\alpha^{k}+1\right)$ and $k=2.533$.

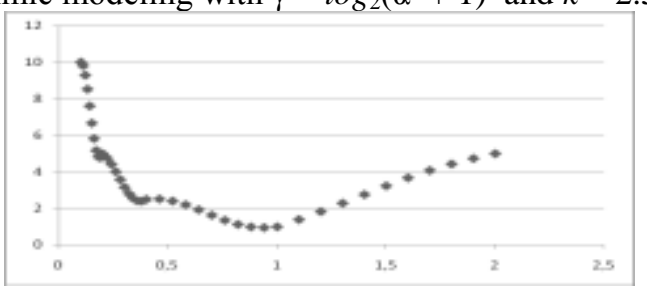

Figure 5. Logarithmic modeling with nine reconstructed points between nodes

These four examples demonstrate possibilities of mathematical 2D process modeling for data nodes. Reconstructed values and interpolated points, calculated by MHR method, are applied in the process of curve modeling for process analysis.

\section{Conclusions}

The method of Hurwitz-Radon Matrices (MHR) enables 2D process modeling using different coefficients $\gamma$ : sinusoidal, cosinusoidal, tangent, logarithmic, exponential, arc sin, arc $\cos$, arc tan or power function [16] or others. Function for $\gamma$ calculations is chosen individually at each $2 \mathrm{D}$ interpolation and depends on initial requirements and data specifications. MHR method leads to 2D mathematical modeling via the set of characteristic points. So MHR makes possible the combination of two important problems: interpolation and modeling. Main features of MHR method are:

a) modeling of $L$ points is connected with the computational cost of rank $O(L)$;

b) MHR is well-conditioned method (orthogonal matrices);

c) coefficient $\gamma$ is crucial in the process of 2D process modeling and it is computed individually for each data.

Future works are going to describe the choice of coefficient $\gamma$ and the features of $2 \mathrm{D}$ process modeling.

\section{References}

[1] Mahoor, M.H., Abdel-Mottaleb, M., Nasser Ansari, A.: Improved Active Shape Model for Facial Feature Extraction in Color Images. Journal of Multimedia 1(4), 21-28 (2006)

[2] Cootes, T.F., Taylor, C.J., Cooper, D.H., Graham, J.: Active Shape Models-Their Training and Application. Computer Vision and Image Understanding 1(61), 38-59 (1995)
[3] Amanatiadis, A., Kaburlasos, V.G., Gasteratos, A., Papadakis, S.E.: Evaluation of Shape Descriptors for Shape-Based Image Retrieval. IET Image Processing 5(5), 493-499 (2011)

[4] Zhang, D., Lu, G.: Review of Shape Representation and Description Techniques. Pattern Recognition 1(37), 1-19 (2004)

[5] Schumaker, L.L.: Spline Functions: Basic Theory. Cambridge Mathematical Library (2007)

[6] Dahlquist, G., Bjoerck, A.: Numerical Methods. Prentice Hall, New York (1974)

[7] Eckmann, B.: Topology, Algebra, Analysis- Relations and Missing Links. Notices of the American Mathematical Society 5(46), 520-527 (1999)

[8] Citko, W., Jakóbczak, D., Sieńko, W.: On Hurwitz - Radon Matrices Based Signal Processing. Workshop Signal Processing at Poznan University of Technology (2005)

[9] Tarokh, V., Jafarkhani, H., Calderbank, R.: Space-Time Block Codes from Orthogonal Designs. IEEE Transactions on Information Theory 5(45), 1456-1467 (1999)

[10] Sieńko, W., Citko, W., Wilamowski, B.: Hamiltonian Neural Nets as a Universal Signal Processor. $28^{\text {th }}$ Annual Conference of the IEEE Industrial Electronics Society IECON (2002)

[11] Sieńko, W., Citko, W.: Hamiltonian Neural Net Based Signal Processing. The International Conference on Signal and Electronic System ICSES (2002)

[12] Jakóbczak, D.: 2D and 3D Image Modeling Using Hurwitz-Radon Matrices. Polish Journal of Environmental Studies 4A(16), 104-107 (2007)

[13] Jakóbczak, D.: Shape Representation and Shape Coefficients via Method of Hurwitz-Radon Matrices. Lecture Notes in Computer Science 6374 (Computer Vision and Graphics: Proc. ICCVG 2010, Part I), SpringerVerlag Berlin Heidelberg, 411-419 (2010)

[14] Jakóbczak, D.: Curve Interpolation Using Hurwitz-Radon Matrices. Polish Journal of Environmental Studies 3B(18), 126-130 (2009)

[15] Jakóbczak, D.: Application of Hurwitz-Radon Matrices in Shape Representation. In: Banaszak, Z., Świć, A. (eds.) Applied Computer Science: Modelling of Production Processes 1(6), pp. 63-74. Lublin University of Technology Press, Lublin (2010)

[16] Jakóbczak, D.: Object Modeling Using Method of Hurwitz-Radon Matrices of Rank k. In: Wolski, W., Borawski, M. (eds.) Computer Graphics: Selected Issues, pp. 79-90. University of Szczecin Press, Szczecin (2010)

About Author:

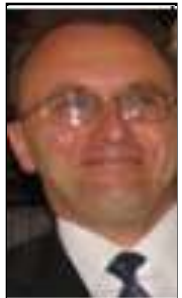

His research interests connect mathematics with computer science and include computer vision, curve interpolation, contour reconstruction and geometric modeling, probabilistic methods and numerical methods. 